or sodium cinnamate was employed in a 1-per-cent. aqueous solution, of which $0.5 \mathrm{grm}$. was at first injected every second day, cocaine being first injected. This was used in various corneal affections, herpes, wounds, pannus, interstitial keratitis, as well as in choroiditis and scleritis. This solution was less painful than the mercurial preparations, and seemed to influence favourably the course of the disease in herpes and interstitial keratitis. It was used persistently in two cases of tuberculous disease of the iris and choroid, but apparently without the slightest effect. $\mathrm{He}$ sums up by saying: My own conclusions, based on observations of my own cases and a careful study of the literature of the subject, are, that all reports of the beneficial effects of subconjunctival injections should be carefully criticised, and compared with the results obtained by other methods of treatment, before accepting them as of any real value.

The Need of a Supplementary Iantern Test for Proper Examination of Colour Vision.-Charles H. Williams ${ }^{5}$ thinks that a very extended use of the Holmgren test in the hands of many examiners is now showing that some cases are able to pass this test with skeins of coloured worsted correctly and without hesitation, who, when examined with the lights from a distant signal, may be unable to distinguish the red and green with any reasunable degree of certainty, and, in fact, often confuse these colours. This he attributes most frequently to cases of acquired defect in colour-vision caused by alcoholtobacco amblyopia. As is well known, this trouble begins only with small scotomata for red and green, and Williams thinks the skeins of wool with which the test is made are large enough to cover the scotoma and a good portion of the surrounding retina, so that its colour is readily recognised. He advocates a test with lights of varying sizes, and also - and it seems to us most important-of varying intensity; this latter of course to correspond with the lights seen through fog, rain, or driving snow, or through steam issuing from the funnels of engines. Williams has devised an electric lamp which carries out these conditions. It struck us very forcibly that the electric lamp used in ophthalmoscopy and made by Curry and Paxton would, with a suitable cover and discs, be a very efficient method of working this test, and the Board of Trade of this country would be well advised to have such a contrivance in their testing stations.

5 Brit. Mєd. Journ., Sept. 26, 1903. 4 Med. Rec., July 18, 1903. 5 Ibid., Aug. 8, 1903.

\title{
Progress in Abdominal Surgery.
}

The Sensibility of the Peritoneum and Abdominal Organs has been observed by Lennander ${ }^{1}$ during operations carried out wholly or in part under local anæsthesia. The results are tabulated in a manner which will be understood from the following extract : Skin incision, no pain ; ligature of vessels in skin, pain ; forceps on a vessel and nerve, severe pain ; puncture of a distended injected bowel, no pain.

Cleansing the Peritoneum by irrigation is preferred by Roberts ${ }^{2}$ when many small clots and much fluid are present. For many years the relative values of sponging and irrigating for the purpose of cleansing the peritoneum have been discussed, and even now no unanimous decision has been reached. Experience shows that it is practically impossible to cleanse the peritoneum by irrigation owing to the innumerable pouches which shelter much of the material which it is desired to wash away. Exceptionally, however, there occur cases in which irrigation is capable of efficiently cleansing the cavity. Especially is this so if there is plenty of as eptic fluid with very little adherent exudation, as in cases of ectopic gestation, for then it matters comparatively little that some of the fluid should be left behind in the peritoneal cavity. In cases, however, where there is much adherent exudation on the surface of the bowel irrigation is of very little value, for it fails utterly to remove the adherent flakes. In these cases swabbing must be employed, but the area of the peritoneum is so large that to cleanse thoroughly the whole surface is a very tedious task. Fortunately the peritoneum possesses the power of dealing with a certain amount of microbic infection, and no surgeon will maintain that by any method that we possess is it possible to render aseptic the peritoneum when its whole surface has become infected.
The Prevention of Ventral Hernia after Laparotomy depends, according to $\mathrm{Wolff},{ }^{3}$ upon whether there has been a greater or less amount of meteorism in the first few days after operation. $\mathrm{He}$ argues, therefore, that the mechanical pressure of the distended bowel causes the stitches, especially those in the fascia, to yield, either by the knot giving way or by the thread cutting through the tissue; the skin suture heals by first intention, and remains intact. $\mathrm{He}$ therefore only completely closes the wound in cases in which an absolutely certain reactionless result is expected; in other cases he inserts a gauze drain. Hahn replies, and gives a record of 88 laparotomies with suture in three layers without a single ventral hernia, whether meteorism occurred or not. His method is :-(1) Isolated-i.e. interrupted-suture of $(a)$ peritoneum (plus transversalis fascia); $(b)$ aponeuroses of abdominal wall to exclusion of muscles; $(c)$ skin with possibly buried sutures of a well-developed panniculus. (2) The sutures should all be of absolutely sterile silk. (3) Complete healing in of the threads. This means strict asepsis. The skin stitches are removed in about eight days ; the patient, as a rule, is up in two weeks, and wears no binder or belt.

Primary Retroperitoneal Sarcoma, unconnected in origin with any of the solid viscera of that region are, says Williams, ${ }^{4}$ by no means common; and the comparative rarity of records of retroperitoneal sarcoma is illustrated by the fact that in 14,630 tumours collected by Gault, of which 894 were sarcomata, only one case of primary sarcoma of this region is reported. Williams reports an unsuccessful operation on a case. The affection is insidious in its onset, indefinite symptoms of a persistently rebellious and growing interference with the digestive organs are its earliest manifestations. Then come the symptoms due to direct pressure on 
the nerves and blood-vessels deep in the abdominal cavity. Finally, the rapidly.growing mass reveals itself to the eye and hand. The tumour is usually immovable, is uninfluenced by the action of the diaphragm, and can generally be separated from the liver or the spleen by an area of resonance. The position of the hollow viscera relative to the growth is of great importance in making a physical examination. This is demonstrated by a band of tympany crossing the mass, with dulness on percussion everywhere else over its surface. As the tumour increases in size it separates the leaflets of the mesentery and pushes the intestine forward. Thus, if the tumour is to the right side of the abdomen, the ascending colon is displaced forward and rests on the anterior and left side of the mass. When the growth is centrally located the small intestines are arranged irregularly over the surface of the tumour; the transverse colon rarely crosses the surface of a median tumour, only three cases being recorded. These physical signs and symptoms are not characteristic of sarcoma alone, but may be present with other neoplasms occupying the same area, nor do they exclude the presence of morbid growths originating in the solid organs of the abdomen. The average duration of the condition is seven to eight months from the beginning of the initial symptoms up to the fatal ending of the case. As to treatment, laparotomy should be performed for two reasons:(1) Exploratory incision is the only sure method of establishing a correct diagnosis; (2) if the possible presence of a tumour is early recognised, a prompt surgical interference may cure the disease or prolong the life of the patient.

1 Brit. Med. Jour., Nov. 14, 1903, p. 1288. 2 Lancet, Oct. 24, 1903, p. 1180. 5 Glasgow Med. Jour., April, 1903, p. 312. ${ }^{4}$ Amer. Jour. Med. Sci., Aug., 1903, p. 269.

\section{Diseases of the Heart and Circulation.}

Variations in Blood-Pressure-Oliver ${ }^{1}$ gives an account of the physiological variations in the bloodpressure, as determined by the sphygmometer and hæmodynometer. There is a wave-like rise and fall in the arterial pressure produced by each meal lasting from two-and-a-half to four hours. Thus a normal wave rises from 100 to $115 \mathrm{~mm}$. of mercury. During digestion there is dilatation of the splanchnic arterioles, a compensatory reduction of the systemic area of the circulation owing to increased tonus of the arterioles, and an increased output from the heart. The capillary blood-pressure is also considerably raised, whilst the venous pressure falls. The rise in the capillary pressure causes an increased exudation of tissue fluid. When food is taken in there is thus not only a flow of digestive secretions but also a concurrent exudation into every tissue of nourishing fluid. The primary effect of exercise is to raise the arterial pressure, but this is only temporary, and it is soon succeeded by a marked fall which continues during the exercise and for some time afterwards. There is thus a large volume of arterial blood in the capillaries, and an increased supply of oxygen to the tissues. In some cases of arterial hypertonus both the capillary and venous pressures are lowered together showing the predominence of arteriolar contraction, and in these cases the hands and feet are apt to be cold and clammy, and the pulse is small. But the heart is hypertrophied and the second aortic sound accentuated. The trouble is really peripheral, and the treatment should be directed to produce dilatation of the arterioles. Tension or static muscular exercises are of great value, and patients find they make the extremities warm. Such exercises are much more beneficial than the application of warmth. The application of external cold, e.g, a cold atmosphere, increases the arteriole contraction and raises the blood pressure, thus putting some strain on the heart. Patients suffering from arterial hypertonus need a warm winter climate as much as a bronchitic individual. Altitude-up to about 3,000 feet-lowers the blood pressure, above that height the pressure rises and the pulse is slightly quicker. This affords an explanation of the facts that great heights are bad for patients with arterial hypertonus, poor hearts or Bright's disease.

Angina Pectoris.-Colbeck has put forward a theory in explanation of the symptoms of angina pectoris. He first observes that the theory of muscular cramp does not explain the phenomena, for, in the first place, there is no physiological evidence that the cardiac muscle can pass into a state of cramp like the skeletal muscles ; again, even if it could take place it would not account for the fact that during the attack the cardiac action still goes on. His theory depends on the well-known fact that disease of the coronary arteries and of the myocardium is in some way associated causally with the incidence of angina pectoris. The characteristic of these myocardial changes is that they are irregularly distributed over the substance of the cardiac walls. These degenerated areas are unable to take their proper share in resisting the increased intra-cardiac pressure, and in consequence undergo more or less distension and stretching. The pain experienced is due to this. The ventricular systole takes place as usual. Hence, as is well known, the action of the heart and the rhythm and character of the heart may remain, as far as physical examination is concerned, undisturbed during the paroxysm. The establishment of mitral regurgitation relieves the pain by lessening the intra-ventricular pressure, and vaso-dilators act similarly. The sense of impending death - the angor animi-is explained thus : if distension of localised patches in the ventricular wall takes place during systole, these areas are simultaneously undergoing contraction and expansion, and send antagonistic impulses to the cardiac centre which is thus thrown into a turmoil. The perturbation of the cardiac: spreads to adjacent centres, and herein lies the explanation of the vaso-motor phenomena as well as of the nausea, vomiting, flatulence and hiccough, which so frequently accompany the paroxysm. Angina. sine dolore is explained on the assumption that the deterioration of the nervous elements in the cardiac: wall has become so great that they fail to react to. stimuli which ordinarily give rise to the sensation of pain.

Infective Endocarditis.-In the Lumleian lectures 